\title{
DISTANCE TO INVERTIBLE LINEAR OPERATORS WITHOUT SEPARABILITY
}

\author{
RICHARD BOULDIN
}

(Communicated by Palle E. T. Jorgensen)

\begin{abstract}
The formula for the distance from a given operator to the invertible operators on a separable Hilbert space is not true if the underlying Hilbert space is not required to be separable. This paper obtains inequalities for that distance in the latter situation. This requires a new concept called the modulus of invertibility, and further study of the concepts of essential nullity and essential deficiency, which permitted us to characterize the closure of the invertible operators.
\end{abstract}

\section{INTRODUCTION}

Extensive results related to the Calkin algebra and operator approximations have been proved for a (bounded linear) operator $T$ on a separable Hilbert space. See [1-5, 9-11], for example. It would be very desirable to have these results on more general spaces, and an important step in that direction is the discovery of appropriate versions for Hilbert spaces that are not necessarily separable. There has been progress toward this goal, as indicated by $[6,8,12]$. The purpose of this paper is to generalize results in $[1,2]$ using some ideas introduced in [6]. We shall introduce a new quantity, denoted $\rho(T)$, which we call the modulus of invertibility.

Let $\mathscr{B}(H)$ denote the operators on the Hilbert space $H$, and define $\operatorname{nul} T(\operatorname{def} T)$ to be the cardinal number $\operatorname{dim} \operatorname{ker} T\left(\operatorname{dim} \operatorname{ker} T^{*}\right)$. Let $U|T|$ be the usual polar factorization of $T$, and let $E(\cdot)$ be the spectral measure for the nonnegative operator $|T|$. In [6] we defined ess nul $T$ by the equation

$$
\text { ess nul } T=\inf \{\operatorname{dim} E([0, \varepsilon)) H: \varepsilon>0\},
$$

and by definition ess def $T$ is ess nul $T^{*}$. In [6] we showed that the closure of the invertible operators is the set of operators $T$ such that ess nul $T=\operatorname{ess} \operatorname{def} T$.

The essential spectrum of $T$, denoted $\sigma_{e}(T)$, is the set $\{z: T-z I$ is not a Fredholm operator $\}$. Define the essential minimum modulus $m_{e}(T)$ to be $\inf \left\{\lambda: \lambda \in \sigma_{e}(|T|)\right\}$. This quantity was studied in [2] for $H$ a separable Hilbert space; it was proved that the distance from $T$ to the set of invertible operators is

Received by the editors October 20, 1990 and, in revised form, March 25, 1991.

1991 Mathematics Subject Classification. Primary 47A05, 47A30; Secondary 47A55.

Key words and phrases. Invertible operator, ring of operators, nonseparable Hilbert space, essential nullity, essential deficiency, modulus of invertibility. 
$\max \left\{m_{e}(T), m_{e}\left(T^{*}\right)\right\}$ provided nul $T \neq \operatorname{def} T$. If nul $T=\operatorname{def} T$ the distance is zero, and it was noted that if both $m_{e}(T)$ and $m_{e}\left(T^{*}\right)$ are positive then they are equal.

For the sake of this current work we define the modulus of invertibility, denoted $\rho(T)$, by the equation

$$
\rho(T)=\inf \{\lambda: \operatorname{dim} E((\lambda-\varepsilon, \lambda+\varepsilon)) H=\operatorname{dim} H \text { for } \varepsilon>0\} .
$$

It is routine to verify that $\rho(T)$ belongs to the set on the right of the preceding formula. When the Hilbert space $H$ is not required to be separable then both of the quantities $m_{e}(T)$ and $\rho(T)$ are required; if $H$ is separable then the two quantities coincide.

\section{BASIC RESUlts}

We need to establish some of the basic properties of $\rho(T)$ and $\rho\left(T^{*}\right)$.

Theorem. (i) $\rho(T) \geq m_{e}(T) \geq 0$.

(ii) Let $G(\cdot)$ denote the spectral measure for $T^{*} T$. The following formula, which is more accessible for computations, is equivalent to the above definition of $\rho(T)$ :

$$
\rho(T)^{2}=\inf \{\lambda: \operatorname{dim} G([0, \lambda+\varepsilon)) H=\operatorname{dim} H \text { for } \varepsilon>0\} .
$$

(iii) If $\rho(T)$ is positive then $\operatorname{nul} T<\operatorname{dim} H$ and $\operatorname{dim} T H=\operatorname{dim} H$.

(iv) We have $\rho(T)=0$ if and only if ess nul $T=\operatorname{dim} H$. Thus, $\rho(T)=0=$ $\rho\left(T^{*}\right)$ implies that $T$ belongs to the closure of the invertible operators, denoted $\mathscr{G}^{-}$.

(v) We have $\rho(T)>0$ if and only if there are operators $B$ and $C$ such that $B T=I+C$ where $\operatorname{dim} C H<\operatorname{dim} H$. Furthermore, $B$ can be chosen so that $\|B\|$ is arbitrarily close to $1 / \rho(T)$.

(vi) We have $\rho\left(T^{*}\right)>0$ if and only if there are operators $B$ and $C$ such that $T B=I+C$ where $\operatorname{dim} C^{*} H<\operatorname{dim} H$.

(vii) If both $\rho(T)$ and $\rho\left(T^{*}\right)$ are positive then they are equal.

Proof. Property (i) follows from the definitions and a standard characterization of the Fredholm spectrum. Property (ii) follows from the spectral mapping theorem applied to $\sqrt{x}$ and the spectral representation of $|T|$ in terms of $G(\cdot)$.

Clearly, if $\operatorname{nul} T=\operatorname{dim} H$ then $\operatorname{nul}|T|=\operatorname{dim} H$ and $\rho(T)=0$. This proves the first assertion in (iii). Since $\operatorname{dim} H=\operatorname{nul} T+\operatorname{dim}(\operatorname{ker} T)^{\perp}$ and $\operatorname{dim}(\operatorname{ker} T)^{\perp}=\operatorname{dim} T H$, the last part of (iii) follows from what is already proved.

A review of the relevant definitions will make the first statement of (iv) clear. Thus, $\rho(T)=0=\rho\left(T^{*}\right)$ implies that ess $\operatorname{nul} T=\operatorname{dim} H=\operatorname{ess} \operatorname{def} T$. The main theorem of [6], as reviewed in the introduction, implies the desired conclusion.

Suppose $\rho(T)>0,0<\varepsilon<\rho(T)$, and let $H_{0}$ denote the subspace $E((\rho(T)-\varepsilon, \infty)) H$. The minimum modulus of $|T|$, and hence $T$ also, restricted to $H_{0}$ is no less than $\rho(T)-\varepsilon$. (See Theorem 1 of [2] for the basic facts about the minimum modulus.) Denote the restriction of $T$ to $H_{0}$, by $T \mid H_{0}$. Define $B$ to be zero on $\left(T H_{0}\right)^{\perp}$, and on $T H_{0}$ let it be the inverse of $T \mid H_{0}$. Thus, $B T \mid H_{0}$ agrees with $I \mid H_{0}$ and $\|B\| \leq 1 /(\rho(T)-\varepsilon)$. Define $C$ by 
$C=I-B T$ and note that

$$
\operatorname{dim} C H=\operatorname{dim} C\left(H_{0}\right)^{\perp} \leq \operatorname{dim}\left(H_{0}\right)^{\perp}<\operatorname{dim} H .
$$

This proves the "only if" part of $(v)$.

Suppose that $B T=I+C$ as in $(\mathrm{v})$, and for the sake of a contradiction assume $\rho(T)=0$. Let positive $\varepsilon$ be given and let $H_{e}=E([0, \varepsilon)) H$; since $\rho(T)=0$, it follows that $\operatorname{dim} H_{\varepsilon}=\operatorname{dim} H$. Because $\operatorname{dim} C H<\operatorname{dim} H$, we know that $\operatorname{dim}(\operatorname{ker} C)^{\perp}<\operatorname{dim} H$ and it follows that there exists a unit vector $g$ in $H_{\varepsilon}$ that is orthogonal to $(\operatorname{ker} C)^{\perp}$. Thus, $\|B T g\|=\|(I+C) g\|=\|g\|=1$ and $\|B T g\|<\|B\| \varepsilon$. By the arbitrariness of $\varepsilon$ we obtain a contradiction, which proves the "if" part of $(\mathrm{v})$.

Part (vi) follows from (v) by taking adjoints.

Note that $T T^{*}=U|T|^{2} U^{*}$, and let $V$ denote the isometry obtained by restricting $U$ to $\left(T^{*} H\right)^{-}$and considering $(T H)^{-}$to be the range of $V$. Since the square root of an operator is a limit of polynomials in the operator, we see that $V|T| V^{*}=\left|T^{*}\right|$ holds on the appropriate subspace. Note that

$$
\left|T^{*}\right|=V\left(\int_{\mathscr{I}} t d E(t)\right) V^{*}=\int_{\mathscr{I}} t d V E(t) V^{*}
$$

where $\mathscr{I}$ is the interval $[0, \infty)$. Since the preceding equation characterizes the spectral measure of $\left|T^{*}\right|$, which we denote $F(\cdot)$, we see that $F(\mathscr{I})=$ $V E(\mathscr{I}) V^{*}$ whenever $\mathscr{I}$ is a subinterval of $(0, \infty)$. If both $\rho(T)=\rho\left(T^{*}\right)$ are positive, then

$$
\begin{gathered}
F\left(\left(\rho\left(T^{*}\right)-\varepsilon, \rho\left(T^{*}\right)+\varepsilon\right)\right)=V E\left(\left(\rho\left(T^{*}\right)-\varepsilon, \rho\left(T^{*}\right)+\varepsilon\right)\right) V^{*}, \\
F((\rho(T)-\varepsilon, \rho(T)+\varepsilon))=V E((\rho(T)-\varepsilon, \rho(T)+\varepsilon)) V^{*} .
\end{gathered}
$$

It follows that $\rho(T)=\rho\left(T^{*}\right)$. This completes the proof of the theorem.

\section{DistANCE TO THE INVERTIBLE OPERATORS}

We are now prepared to obtain inequalities for the distance from a given operator $T$ to the set of invertible operators $\mathscr{G}$.

Theorem 2. If ess nul $T=$ ess def $T$, then $\operatorname{dist}(T, \mathscr{G})=0$. If ess nul $T \neq$ ess def $T$, then we have

$$
\operatorname{dist}(T, \mathscr{G}) \leq \max \left\{\rho(T), \rho\left(T^{*}\right)\right\},
$$

which implies that not both $\rho(T)$ and $\rho\left(T^{*}\right)$ are zero.

Proof. The first statement is Theorem 3 of [6]. In view of Theorem 1(iv) not both quantities $\rho(T)$ and $\rho\left(T^{*}\right)$ are zero. Interchange $T$ and $T^{*}$, if necessary, so that we may assume that $\rho(T)>0$. Let $\mu$ denote $\rho(T)$, let positive $\varepsilon$ be given, and let $U R$ be the polar factorization of $T$ such that $\operatorname{ker} U=\operatorname{ker} R$. Thus,

$$
\operatorname{dim} U E((0, \mu+\varepsilon)) H=\operatorname{dim} E((0, \mu+\varepsilon)) H=\operatorname{dim} H .
$$

Define $R(\varepsilon)$ to be $R$ on $E([\mu+\varepsilon, \infty)) H$ and $\mu I$ on $E([0, \mu+\varepsilon)) H$. Define $U(\varepsilon)$ to be $U$ on $E([\mu+\varepsilon, \infty)) H$ and on $E([0, \mu+\varepsilon)) H$ let it be $\varepsilon V$, where $V$ is an isometry of $E([0, \mu+\varepsilon)) H$ onto $\operatorname{span}\left\{U E((0, \mu+\varepsilon)) H,(T H)^{\perp}\right\}$. Since

$$
U E((0, \infty)) H=U(R H)^{-}=(T H)^{-},
$$


it is clear that $U(\varepsilon)$ is one-to-one and onto; thus, $U(\varepsilon)$ is invertible. Clearly $R(\varepsilon)$ is invertible and

$$
\begin{aligned}
\|U R-U(\varepsilon) R(\varepsilon)\| \leq & \|(U-U(\varepsilon)) R \mid E([0, \mu+\varepsilon)) H\| \\
& +\|U(\varepsilon)(R-R(\varepsilon)) \mid E([0, \mu+\varepsilon)) H\| \\
& \leq \mu+3 \varepsilon \mu+\varepsilon+2 \varepsilon^{2} .
\end{aligned}
$$

This proves that $\operatorname{dist}(T, \mathscr{G})$ is not more than $\mu$. It follows from Theorem 1 (vii) that $\mu=\max \left\{\rho(T), \rho\left(T^{*}\right)\right\}$.

In [2] when we were proving a similar result for operators on a separable Hilbert space, we used a folklore theorem to deduce the inequality opposite to the one given in Theorem 2. That folklore theorem asserts that $\|A-B\|<m_{e}(A)$ implies that ind $A=$ ind $B$, where ind $T$ denotes the index of $T$; i.e., ind $T=$ nul $T$ - def $T$. Unfortunately, there is no result for nonseparable Hilbert spaces analogous to the folklore theorem. Because the range of a compact operator cannot contain a nonseparable subspace, the theory for compact perturbations is intrinsically inadequate. See Theorem 6 of [6].

In order to prove an inequality opposite to the one in Theorem 2 , we must use an elaborate constructive technique. Provided that $\rho(T)>0$, we define $\eta(T)$ by

$$
\eta(T)=\sup \{\lambda: \operatorname{dim} E([0, \lambda)) H=\operatorname{ess} \operatorname{nul} T \text { and } \lambda \leq \rho(T)\} .
$$

If $\rho(T)=0$ then we define $\eta(T)=0$. We note that $\rho(T)=0$ implies that ess nul $T=\operatorname{dim} H$ by Theorem 1 (iv). Thus, we informally regard $\eta(T)$ as a point where the rank of the resolution of identity jumps from ess nul $T$ to a larger cardinal number. For positive $\varepsilon$ the function $\operatorname{dim} E([0, \varepsilon)) H$ is a nondecreasing function taking values in the cardinal numbers. If this function did not assume the value ess nul $T$ then the successor of that cardinal number would be a larger lower bound. Thus, ess nul $T$ is a value assumed by $\operatorname{dim} E([0, \varepsilon)) H$ for all $\varepsilon$ in some interval $(0, \delta)$. It follows that $\eta(T)$ is positive unless $\rho(T)=0$.

Whenever $\eta(T)$ is positive then it is characterized by the formulas

$$
\operatorname{dim} E([0, \varepsilon)) H=\operatorname{ess} \text { nul } T \text { provided } 0<\varepsilon<\eta(T)
$$

and

$$
\operatorname{dim} E([0, \delta+\eta(T))) H>\text { ess nul } T \text { for } \delta>0 .
$$

If the $\varepsilon$ is the first formula is replaced by $\eta(T)$ then the equation may fail to be true depending on whether $\eta(T)$ is a limit cardinal or not. The preceding observations will be used throughout the paper.

Lemma 3. If ess nul $T<$ ess def $T$ then $\eta(T) \leq \eta\left(T^{*}\right)$.

Proof. By Lemma 2 of [6] we know that $U E(\mathscr{J})=F(\mathscr{J}) U$ for any interval $\mathscr{I} \subset(0, \infty)$ where $F(\cdot)$ and $E(\cdot)$ are the spectral measures of $\left|T^{*}\right|$ and $|T|$, respectively, and $U|T|$ is the polar factorization of $T$. The hypothesis implies that ess nul $T<\operatorname{dim} H$ and Theorem 1(iv) shows that $\rho(T)>0$. The remarks preceding this lemma show that $\eta(T)>0$.

It is elementary and routine to show that $T$ has closed range if and only if $T^{*}$ does. Since $U$ is a linear isometry that maps the closure of $|T| H$, denoted $(|T| H)^{-}$, onto $(T H)^{-}$, we see that either all of the operators $|T|, T^{*}, T,\left|T^{*}\right|$ have closed range or none does. It is elementary and routine to show that 
$|T| H$ (or $\left|T^{*}\right| H$ ) is closed if and only if 0 is not an accumulation point of the spectrum of $|T|$ (or $\left.\left|T^{*}\right|\right)$, denoted $\sigma(|T|)$ (or $\sigma\left(\left|T^{*}\right|\right)$ ). It now follows that if $T H$ is closed then ess nul $T=\operatorname{nul} T$ and ess $\operatorname{def} T=\operatorname{def} T$.

For the sake of brevity we let $\alpha=\eta(T)$ and $\beta=\eta\left(T^{*}\right)$. We prove the assertion of the lemma in the case that $T H$ is closed. We see that

$$
\operatorname{dim} F(\{0\}) H=\operatorname{def} T=\operatorname{ess} \operatorname{def} T>\operatorname{ess} \operatorname{nul} T=\operatorname{nul} T=\operatorname{dim} E(\{0\}),
$$

and according to the first paragraph,

$$
\operatorname{dim} F((0, \varepsilon)) H=\operatorname{dim} E((0, \varepsilon)) H \quad \text { for } \varepsilon>0 .
$$

Thus, for $0<\varepsilon<\beta$ and $0<\delta$

$$
\operatorname{dim} E([0, \varepsilon)) H<\operatorname{dim} F([0, \varepsilon)) H=\operatorname{ess} \operatorname{def} T
$$

and

$$
\operatorname{dim} E([\varepsilon, \beta+\delta)) H=\operatorname{dim} F([\varepsilon, \beta+\delta)) H>0 .
$$

If ess def $T$ is finite then so is ess nul $T$ and the above formulas show that $\operatorname{dim} E([0, \gamma)) H$ jumps at $\gamma=\beta$, which implies that $\alpha \leq \beta$, as defined. If ess def $T$ is infinite then $\operatorname{dim} F([\varepsilon, \beta+\delta)) H$ is a larger cardinal number than $\operatorname{dim} F([0, \varepsilon)) H$ and $\operatorname{dim} E([0, \gamma)) H$ jumps up at $\gamma=\beta$ regardless of the cardinality of ess nul $T$.

If $T H$ is not closed then the second paragraph of this proof shows that 0 is an accumulation point $\sigma(|T|)$ and $\sigma\left(\left|T^{*}\right|\right)$. It follows that ess nul $T$ and ess $\operatorname{def} T$ are infinite cardinal numbers. The formulas $\operatorname{dim} F([0, \varepsilon)) H=\operatorname{ess} \operatorname{def} T$ provided $0<\varepsilon<\beta$ and

$$
\operatorname{dim} F([0, \beta+\delta)) H>\operatorname{ess} \operatorname{def} T \text { for } \delta>0
$$

imply that

$$
\operatorname{dim} F([\varepsilon, \beta+\delta)) H>\text { ess def } T .
$$

Since $\operatorname{dim} E([\varepsilon, \beta+\delta)) H=\operatorname{dim} F([\varepsilon, \beta+\delta)) H$, it follows that $\operatorname{dim} E([0, \gamma)) H$ jumps at $\gamma=\beta$ and that implies $\alpha \leq \beta$, as desired.

Lemma 4. Suppose that ess nul $T \neq \operatorname{ess} \operatorname{def} T$ and let $\mu$ denote the smallest positive number in $\left\{\eta(T), \eta\left(T^{*}\right)\right\}$. If $\|T-B\|<\mu$ then $B$ is not invertible, and so $\operatorname{dist}(T, \mathscr{G}) \geq \mu$.

Proof. Let $B$ be an operator such that $\|T-B\|<\mu$. Interchange $T$ and $T^{*}$, if necessary, so that ess nul $T<\operatorname{ess} \operatorname{def} T$. Since ess $\operatorname{def} T \leq \operatorname{dim} H$, we see that ess nul $T<\operatorname{dim} H$. It follows from Theorem 1 (iv) that $\rho(T)>0$. It follows from Lemma 3 and the remarks preceding that lemma that $\mu=\eta(T)$. Let $H_{0}$ represent $E([\lambda, \infty)) H$ where $\lambda$ is chosen between $\|T-B\|$ and $\mu$, and let $Q$ denote $E([\lambda, \infty))$, which is the orthogonal projection onto $H_{0}$. Construct the polar factorization of $T$, say $U|T|$, such that $\operatorname{ker} U=\left(T^{*} H\right)^{\perp}=\operatorname{ker} T$.

Denote the restriction of $T$ to $H_{0}$ by $T \mid H_{0}$. We have

$$
\begin{gathered}
\left\|Q U^{*}(T-B) \mid H_{0}\right\| \leq\|T-B\|<\lambda, \\
Q U^{*} T\left|H_{0}=Q U^{*} U\right| T|| H_{0}=|T| \mid H_{0} .
\end{gathered}
$$

Since $m\left(|T| \mid H_{0}\right) \geq \lambda$, we can find an inverse for $|T| \mid H_{0}$, which we denote by $C$, such that $\|C\|=1 / m\left(|T| \mid H_{0}\right) \leq 1 / \lambda$. Thus, we have

$$
\left\|\left(I-C Q U^{*} B\right) \mid H_{0}\right\| \leq\|C\|\|T-B\|<1,
$$


and we conclude that $C Q U^{*} B \mid H_{0}$ is invertible. Since $C Q U^{*} B \mid H_{0}$ is onto $H_{0}$, it must be that $Q U^{*} B \mid H_{0}$ is onto as well as one-to-one. It follows that $U Q U^{*} B \mid H_{0}$ is an invertible map of $H_{0}$ onto $U H_{0}$.

By Lemma 2 of [6] we know that $U E(\mathscr{I})=F(\mathscr{I}) U$ for any interval $\mathscr{I} \subset$ $(0, \infty)$ where $F(\cdot)$ and $E(\cdot)$ are the spectral measures of $\left|T^{*}\right|$ and $|T|$, respectively. Recall that $U H=(T H)^{-}=\left(\left|T^{*}\right| H\right)^{-}=F((0, \infty)) H$. Thus,

$$
U H_{0}=F([\lambda, \infty)) U H=F([\lambda, \infty)) H,
$$

and we denote this last space by $H_{1}$. Because of Lemma 2 of [6], we know that

$$
\operatorname{dim} E((0, \varepsilon)) H=\operatorname{dim} F((0, \varepsilon)) H
$$

for positive $\varepsilon$.

We choose a positive number $\gamma$ such that

$$
\operatorname{dim} E([0, \varepsilon)) H=\operatorname{ess} \operatorname{nul} T \text { and } \operatorname{dim} F([0, \varepsilon)) H=\operatorname{ess} \operatorname{def} T
$$

provided $0<\varepsilon \leq \gamma$. We note that

$$
\operatorname{dim} E((0, \varepsilon)) H=\operatorname{dim} F((0, \varepsilon)) H .
$$

Now we consider the case that $T H$ is not closed. It follows from the second paragraph of the proof of Lemma 3 that ess nul $T$ and ess def $T$ are both infinite. We note that

$$
\begin{aligned}
\operatorname{def} T+\operatorname{dim} F((0, \gamma)) H & =\operatorname{dim} F([0, \gamma)) H=\text { ess } \operatorname{def} T>\text { ess nul } T \\
& =\operatorname{dim} E([0, \gamma)) H=\operatorname{nul} T+\operatorname{dim} E((0, \gamma)) H .
\end{aligned}
$$

Since the sum of any set of cardinal numbers that includes an infinite cardinal is simply the maximum of the cardinals, we deduce that ess $\operatorname{def} T=\operatorname{def} T$ and

$$
\text { nul } T \leq \operatorname{ess} \operatorname{nul} T<\operatorname{ess} \operatorname{def} T=\operatorname{def} T .
$$

On the other hand, if $T H$ is closed then the second paragraph in the proof of Lemma 3 implies that nul $T=\operatorname{ess} \operatorname{nul} T$ and $\operatorname{def} T=\operatorname{ess} \operatorname{def} T$. Thus, we again obtain

$$
\text { nul } T \leq \operatorname{ess} \operatorname{nul} T<\operatorname{ess} \operatorname{def} T=\operatorname{def} T .
$$

Since $U U^{*}$ is the orthogonal projection onto $\left(\left|T^{*}\right| H\right)^{-}=(T H)^{-}$, we deduce that $U U^{*}=F((0, \infty))$.

Also, we see that

$$
\begin{aligned}
U Q U^{*} B \mid H_{0} & =F([\lambda, \infty)) U U^{*} B \mid H_{0} \\
& =F([\lambda, \infty)) F((0, \infty)) B\left|H_{0}=F([\lambda, \infty)) B\right| H_{0} .
\end{aligned}
$$

Thus, $F([\lambda, \infty)) B \mid H_{0}$ is an invertible map of $H_{0}$ onto $H_{1}$, and $F([\lambda, \infty))$ is an invertible map of $B H_{0}$ onto $H_{1}$.

For the sake of a contradiction, we assume that $B$ is invertible. Clearly $B H_{0}+B\left(H_{0}\right)^{\perp} \supset B\left(H_{0}+\left(H_{0}\right)^{\perp}\right)=B H=H$ and $B H_{0} \cap B\left(H_{0}\right)^{\perp} \neq\{0\}$ would contradict that $B$ is one-to-one. Thus, $H=B H_{0} \oplus B\left(H_{0}\right)^{\perp}$ (however, the direct sum is not orthogonal unless $B$ is unitary). Clearly the quotient space $H / B H_{0}$ is isomorphic to both $B\left(H_{0}\right)^{\perp}$ and $\left(B H_{0}\right)^{\perp}$; thus, $\operatorname{dim}\left(B H_{0}\right)^{\perp}=\operatorname{dim} B\left(H_{0}\right)^{\perp}$. Since $B$ is one-to-one,

$$
\begin{aligned}
\operatorname{dim} B\left(H_{0}\right)^{\perp} & =\operatorname{dim}\left(H_{0}\right)^{\perp}=\operatorname{dim} E([0, \lambda)) H \\
& =\operatorname{nul} T+\operatorname{dim} E((0, \lambda)) H .
\end{aligned}
$$


Before we can conclude the proof of this lemma, we need to verify one more observation. Since $F([\lambda, \infty))$ maps $B H_{0}$ onto $H_{1}=F([\lambda, \infty)) H$, for any vector $f$ there exists $g \in B H_{0}$ such that

$$
F([\lambda, \infty)) g=F([\lambda, \infty)) f \text { or } f-g \in \operatorname{ker} F([\lambda, \infty)) .
$$

It follows that $H \subset B H_{0}+\operatorname{ker} F([\lambda, \infty))$. Since $F([\lambda, \infty)) \mid B H_{0}$ is one-to-one, the preceding sum must be a direct sum. The decompositions

$$
B H_{0} \oplus \operatorname{ker} F([\lambda, \infty))=H=B H_{0} \oplus\left(B H_{0}\right)^{\perp}
$$

imply that $\operatorname{dim}\left(B H_{0}\right)^{\perp}=\operatorname{nul} F([\lambda, \infty))$.

Now we can conclude the proof by noting that

$$
\begin{aligned}
\operatorname{dim} B\left(H_{0}\right)^{\perp} & =\operatorname{dim}\left(B H_{0}\right)^{\perp}=\operatorname{nul} F([\lambda, \infty))=\operatorname{dim} F([0, \lambda)) H \\
& =\operatorname{def} T+\operatorname{dim} F((0, \lambda)) H=\operatorname{def} T+\operatorname{dim} E((0, \lambda)) H .
\end{aligned}
$$

Since $\lambda<\mu \leq \eta(T)$, we know that $\operatorname{dim} E((0, \lambda)) H=\operatorname{ess}$ nul $T$, and we have already shown that ess nul $T<\operatorname{def} T$. From this and the fact that nul $T \leq$ ess nul $T$, it follows that

$$
\operatorname{def} T+\operatorname{dim} E((0, \lambda)) H>\operatorname{nul} T+\operatorname{dim} E((0, \lambda)) H
$$

independent of whether the cardinal numbers are finite or infinite. If $\operatorname{dim} E((0, \lambda)) H$ is infinite then the left side of the inequality equals $\operatorname{def} T$ and the right side equals $\operatorname{dim} E((0, \lambda)) H$. If $\operatorname{dim} E((0, \lambda)) H$ is finite then the inequality follows from $\operatorname{def} T>\operatorname{nul} T$.

This contradiction proves that $B$ is not invertible and completes the proof of the lemma.

Theorem 5. If ess nul $T=$ ess def $T$ then $\operatorname{dist}(T, \mathscr{G})=0$. If ess nul $T \neq$ ess $\operatorname{def} T$ then we have $\lambda \leq \operatorname{dist}(T, \mathscr{G}) \leq \max \left\{\rho(T), \rho\left(T^{*}\right)\right\}$, where $\lambda$ is the smallest positive number in $\left\{\eta(T), \eta\left(T^{*}\right)\right\}$.

Proof. This follows from Theorem 2 and Lemma 4.

Corollary 6. If ess nul $T \neq$ ess def $T, \eta(T)=\rho(T)$, and $\eta\left(T^{*}\right)=\rho\left(T^{*}\right)$, then

$$
\operatorname{dist}(T, \mathscr{G})=\max \left\{\rho(T), \rho\left(T^{*}\right)\right\} .
$$

Proof. We consider the case that $\eta(T)=\rho(T)>0$, since the other case is analogous. The desired conclusion follows from the equation

$$
\lambda=\eta(T)=\rho(T)=\max \left\{\rho(T), \rho\left(T^{*}\right)\right\} .
$$

Here we have used that $\rho(T)=\rho\left(T^{*}\right)$ when both quantities are positive.

The preceding corollary gives the distance to the invertibles for many classes of operators. For example, if $T$ is a partial isometry with ess nul $T \neq \operatorname{ess} \operatorname{def} T$ then $T T^{*}$ and $T^{*} T$ are projections onto nontrivial subspaces. The spectrum of each of these is either $\{0,1\}$ or $\{1\}$. Thus, $\eta(T)=\rho(T)=1$ and $\eta\left(T^{*}\right)=$ $\rho\left(T^{*}\right)=1$, and the corollary shows that $\operatorname{dist}(T, \mathscr{G})=1$.

\section{Related Results}

In [6] we showed that ess nul $T$ had a lower semicontinuity property analogous to nul $T$ and that ess nul $T$ could be characterized without using the spectral measure for $|T|$. Now we want to exhibit other properties that help to explain our choice of language. These properties should facilitate future applications. 
Theorem 7. Let $T$ and a positive $\varepsilon$ be given. There exists an operator $P$ such that

(i) $\|P\|<\varepsilon$,

(ii) $(T+P)$ has closed range,

(iii) ess nul $(T+P)=\operatorname{nul}(T+P)=\operatorname{ess} \operatorname{nul} T$,

(iv) ess $\operatorname{def}(T+P)=\operatorname{def}(T+P)=\operatorname{ess} \operatorname{def} T$,

(v) $\rho(T+P)=\rho(T)$ provided $\rho(T)>0$.

Proof. Let $Q$ denote the orthogonal projection $E([0, \varepsilon / 2))$, let $P=-T Q$, and let $H_{1}=Q H$. Note that

$$
\|P\|=\|U|T| Q\| \leq\left\||T| \mid H_{1}\right\| \leq \varepsilon / 2,
$$

and

$$
T+P=U|T|(I-Q)=U|T| E([\varepsilon / 2, \infty)) .
$$

Because the origin is not an accumulation point for the spectrum of $|T| E([\varepsilon / 2, \infty))$, this operator has closed range. Since $U$ is isometric on the range of $|T|$, we see that $U|T| E([\varepsilon / 2, \infty))$ has closed range. This proves (i) and (ii).

Revise our choice of $\varepsilon$, if necessary, so that $\operatorname{dim} E([0, \delta)) H$ is the constant ess nul $T$ for $0<\delta \leq \varepsilon / 2$. Clearly the kernel of $(T+P)=U|T| E([\varepsilon / 2, \infty))$ is $H_{1}$ and $\operatorname{nul}(T+P)=$ ess nul $T$. Since $(T+P)$ has closed range, ess nul $(T+P)=$ $\operatorname{nul}(T+P)$. Thus, (iii) is proved.

Let $F(\cdot)$ be the spectral measure for $\left|T^{*}\right|$. Note that

$$
\begin{aligned}
(T+P)(T+P)^{*} & =T(I-Q)^{2} T^{*}=T(I-Q)|T| U^{*} \\
& =T|T| E([\varepsilon / 2, \infty)) U^{*} \\
& =T|T| U^{*} F([\varepsilon / 2, \infty))=\left(T T^{*}\right) F([\varepsilon / 2, \infty)) .
\end{aligned}
$$

This formula makes it clear that $\left|(T+P)^{*}\right|$ has closed range and its kernel is exactly $F([0, \varepsilon / 2)) H$. The remainder of the proof of (iv) is analogous to the preceding paragraph.

If $\rho(T)>0$ then ess nul $T<\operatorname{dim} H$, and we can choose a positive $\varepsilon<\rho(T)$ so that $\operatorname{dim} E([0, \varepsilon)) H=$ ess nul $T$. We use the $P$ that has been previously defined with such an $\varepsilon$. We note that $U^{*} U$ is the projection onto $|T| H$ and so $U^{*} U|T|=|T|$; also, we note that $(I-Q)$ commutes with $|T|$. Thus, we have

$$
\begin{aligned}
|T+P| & =\left((I-Q)|T| U^{*} U|T|(I-Q)\right)^{1 / 2} \\
& =\left((I-Q)|T|^{2}(I-Q)\right)^{1 / 2}=|T|(I-Q) .
\end{aligned}
$$

If $G(\cdot)$ is the spectral measure for $|T+P|$ then $G(\Delta)=E(\Delta \cap[\varepsilon / 2, \infty))$ for any set $\Delta$. Thus, for $\lambda \geq \varepsilon$ and positive $\delta$ sufficiently small we have

$$
\operatorname{dim} G((\lambda-\delta, \lambda+\delta)) H=\operatorname{dim} E((\lambda-\delta, \lambda+\delta)) H,
$$

and it follows that $\rho(T+P)=\rho(T)$. This proves $(\mathrm{v})$ and the theorem.

Remark. After this paper was submitted, the author discovered [7], which takes a different approach to the same problem with a resulting formula. 


\section{REFERENCES}

1. C. Apostol, L. A. Fialkow, D. A. Herrero, and D. Voiculescu, Approximation of Hilbert space operators, Vol. II, Pitman, Boston, MA, 1984.

2. R. H. Bouldin, The essential minimum modulus, Indiana Univ. Math. J. 30 (1981), 513517.

3. 139-143.

4. _ Approximation by operators with fixed nullity, Proc. Amer. Math. Soc. 103 (1988), 141-144.

5. _ Approximation by semi-Fredholm operators with fixed nullity, Rocky Mountain J. Math. 20 (1990), 39-50.

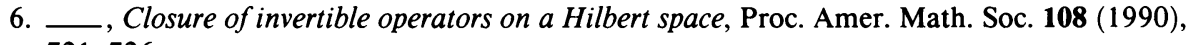
721-726.

7. L. Burlando, Distance formulas on operators whose kernel has fixed Hilbert dimension, Rend. Mat. (7) 10 (1990), 209-238.

8. R. Harte, Regular boundary elements, Proc. Amer. Math. Soc. 99 (1987), 328-330.

9. D. A. Herrero, Approximation of Hilbert space operators, Vol. I, Pitman, Boston, MA, 1982.

10. S. Izumino, Inequalities on operators with index zero, Math. Japonica 23 (1979), 565-572.

11. P. Y. Wu, Approximation by invertible and noninvertible operators, J. Approx. Theory 56 (1989), 267-276.

12. J. Zemánek, Geometric interpretation of the essential minimum modulus, Invariant Subspaces and Other Topics, Birkhäuser Verlag, Basel, 1982, pp. 225-227.

Department of Mathematics, University of Georgia, Athens, Georgia 30602

E-mail address: rbouldin@jce.math.uga.edu 